\title{
Alpha lipoic acid (ala) combining with corticosteroid hormone improves the outcome of infectious diseases in spinal cord ( 10 cases study)
}

\begin{abstract}
Infectious diseases of spinal cord, including Myelitis, Neuromylitis Optica(NMO) and Multiple Sclerosis(MS), are costly progressive and often cause life-time disabilities. Currently, corticosteroid hormone is only routine effective therapy, but serious side effects limit its clinical application. Alpha Lipoic Acid (ALA) is traditional Chinese medicine isolated from natural products such as liver, broccoli, tomatoes and so on. ALA has been used in treatment of degeneration diseases for eyes, diabetics. However, the evidence of anti-inflammation of Multiple Sclerosis (MS) is too less to be conclusive. Here, we present 10 cases that the combination of routine therapeutic effective corticosteroid hormone with ALA improves the outcome of patients with chronic and acute infectious diseases in spinal cord, corresponding to the reversed damage lesions in Magnetic Resonance Imaging (MRI) image and improvement of Extended Disability Status Scale (EDSS) scores with the time of diseases course.
\end{abstract}

Keywords: alpha lipoic acid (ALA), corticosteroid hormone, myelitis, neuromylitis optica(NMO), multiple sclerosis(MS), extended disability status scale (EDSS), magnetic resonance imaging (MRI) image, brain blood barrier (BBB)

\author{
Volume 7 Issue 5 - 2017
}

\author{
Xin Wang,' Anne Hubbard,' Guojun Tan² \\ 'ACURE Biotechnology, INC. Ellicott City, USA \\ 2Department of Neurology, The Second Hospital of Hebei \\ Medical University, China
}

Correspondence: Xin Wang, Acure Biotechnology, INC Ellicott City, MD 2143, USA, Email xwang354@gmail.com

Received: August 02, 2017 | Published: October 23, 2017

\section{Introduction}

Myelitis, Neuromylitis Optica (NMO) and Multiple Sclerosis (MS) are clinic common infectious diseases in spinal cord that are costly, progressive and often cause life-time disabilities. Methylprednisolone Sodium Succinate, a corticosteroid hormone, is a routine effective therapeutic drug for center nerve system (CNS) inflammation, especially applied for MS attacks. ${ }^{1}$ However, long term and/or high dose use of corticosteroid hormone has serious side effects such as osteonecrosis of femoral head, cerebral hemorrhage, unstable blood pressure and blood sugar, disorder of kidney. ${ }^{2}$ Therefore, corticosteroid hormone therapy is limited on clinical application.

The Alpha Lipoic Acid (ALA) is traditional Chinese medicine isolated from natural products such as liver, broccoli, tomatoes and so on. ${ }^{3}$ ALA has been used in treatment of degeneration diseases for eyes, ${ }^{4}$ diabetic polyneuropathies, ${ }^{5}$ vascular diseases as anti-oxidant/ anti-aging therapy. ${ }^{6,7}$ It showed compromising results in treatment of MS with animal models, however, anti-inflammatory properties of ALA in pilot clinical trials have rarely been investigated and only with controversial evidence. ${ }^{8,9}$ Here, we present the evidence in 10 cases that the combination of corticosteroid hormone and ALA clearly improve the EDSS scores of chronic and acute inflammation diseases in spinal cord of patients, corresponding to the reversed damage lesions in MRI image.

\section{Methods and materials}

The clinical subjects in this study are 10 inpatients (age 14-66 years old, female9/ male1) with acute or chronic repeated inflammation on spinal cord. Among them, one case is first onset of acute myelitis; nine cases are acute onset of 5 cases of MS and 4 cases of NMO. The chronic disease course is from 2 months to 7 years. Only one patient in this study had relapsed in six months.

The protocol of the clinic study is to give high dose $500 \mathrm{mg} /$ day i.v. of Methylprednisolone Sodium Succinate as a routine treatment at the time point of becoming inpatient, to gradually reduce half of dose of Methylprednisolone Sodium Succinate every 3 days until removal of this hormone. ALA is applied 300mg twice a day as early as possible and maintains to the end of this study. The EDSS scores are accessed at the first day of taking ALA (Initial), then at one month, three month and six month of taking ALA. MRI examination are carried out at onset of diseases, one month, three month and one year after taking ALA in both sections of median sagittal and transverse (Figure 1A). This protocol has approval from the clinical research ethic committee of the Second Hospital of Hebei Medical University. All patients signed informed consent forms.

Statistics analyses are performed with R Software. All EDSS scores are included in coordinates that Y-axis is EDSS score and $\mathrm{X}$-axis is time points of treatment. The human subjects data are analyzed with BOXes with mini, maxi and mean values. The first quarter, the median, the third quarter and the mini, the maxi, the mean values are summarized in Table 1.

Table I The statistic analysis of changes of Expanded Disability Status Scale (EDSS) of patients with acute myelitis, acute onset of MS and NMO $(n=10)$ at different time points after ALA treatment

\begin{tabular}{|c|c|c|c|c|c|c|}
\hline & Min & \multicolumn{2}{|c|}{ Ist Qu. Median } & $3^{\text {rd }} Q_{u}$ & Max & Mean \\
\hline Initial & 1.5 & 2.5 & 4.5 & 5.875 & 7.5 & 4.35 \\
\hline$\left.\right|^{\text {st }}$ Month & 0 & 2 & 4.25 & 5.25 & 6 & 3.55 \\
\hline $3^{\text {rd }}$ Month & 0 & 1.128 & 2.25 & 4.75 & 5.5 & 2.701 \\
\hline $6^{\text {th }}$ Month & 0 & 0 & 1.75 & 3.875 & 5 & 2.05 \\
\hline
\end{tabular}

\section{Results}

Figure 1A shows that Alpha Lipoic Acid (ALA) combining with Methylprednisolone Sodium Succinate improves the outcome of acute and chronic patients with inflammation in spinal cord with the examination of Magnetic Resonance Imaging (MRI) showing the changes of inflammatory lesions in cervical cord of a NMO patient through disease course in one year under treatment of ALA. When acute onset of MS, there is extensive abnormal enhanced long T1W1, 
long $\mathrm{T} 2 \mathrm{~W} 1$ signal from $\mathrm{C} 1$ to $\mathrm{C} 6$ spine cord levels in median sagittal section. The transverse section between $\mathrm{C} 3$ and $\mathrm{C} 4$ shows that the lesion is mainly around central canal and posterior funiculus. The following up examination of MRI after three months shows the area of lesion is significantly smaller, the extensive abnormal enhanced long $\mathrm{T} 1 \mathrm{~W} 1$, long $\mathrm{T} 2 \mathrm{~W} 1$ signal is limited from $\mathrm{C} 1$ to $\mathrm{C} 4$ spine cord levels in median sagittal section. The transverse section between $\mathrm{C} 3$ and $\mathrm{C} 4$ shows the flaky signal foci lesion at posterior funiculus. The following up examination of MRI after six months shows the area of lesion is more smaller, the extensive abnormal linear long T1W1, long T2W1 signal is limited from $\mathrm{C} 1$ to $\mathrm{C} 4$ spine cord levels in median sagittal section. The transverse section between $\mathrm{C} 3$ and $\mathrm{C} 4$ shows the spotted and flaky signal foci lesion at posterior funiculus. The following up examination of MRI at one year after taking ALA shows the area of lesion is much more smaller, the extensive abnormal enhanced long T1W1, long T2W1 signal is limited from $\mathrm{C} 1$ to $\mathrm{C} 3$ spine cord levels in median sagittal section. The transverse section between $\mathrm{C} 3$ and $\mathrm{C} 4$ shows the spotted signal foci in posterior funiculus area. These image results indicate that ALA treatment reduces the inflammation lesions in spinal cord of MS patient.

\section{A}

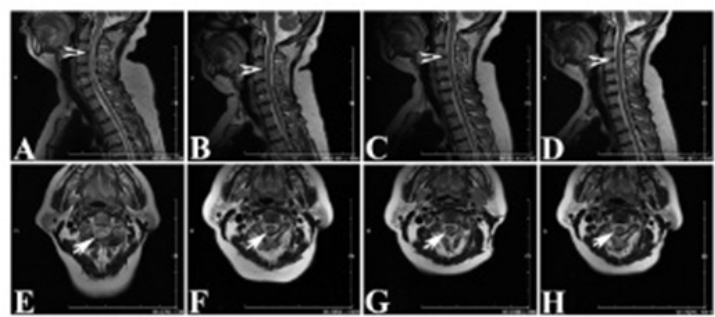

Figure IA ALA combining with routine corticosteroid therapy reverses inflammation legions in MRI image of a patient with NMO through disease course in one year under treatment of ALA .A-D are median sagittal sections following time points of disease course (accordingly onset, three month, six month and one year), arrowheads indicate the level of transverse section of lower line $(\mathrm{E}-\mathrm{H})$ that are between $\mathrm{C} 3$ and $\mathrm{C} 4 \mathrm{E}-\mathrm{H}$ are transverse sections of indication on upper line (A-D). Arrows show inflammation legions of spinal cord.
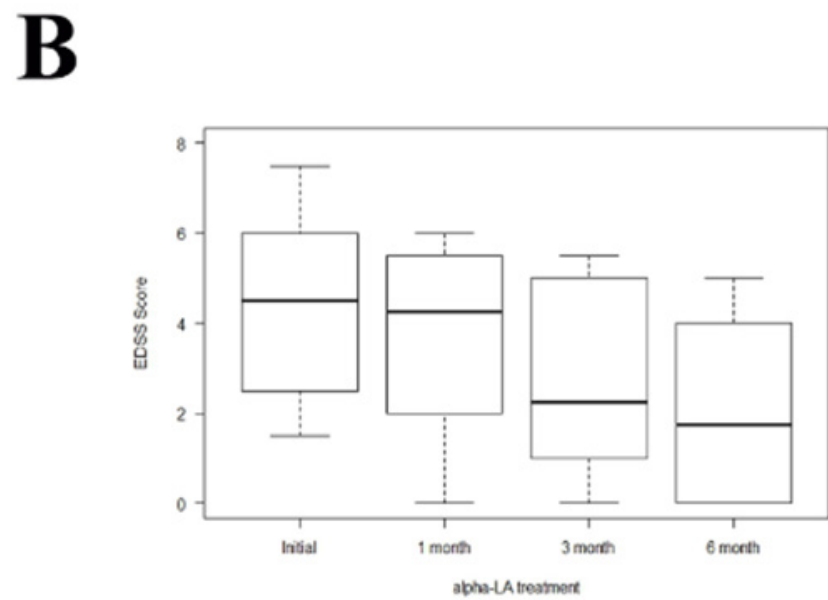

Figure IB BOXes analysis of EDSS scores from 10 cases patients with acute or chronic inflammation in spinal cord following the time points of disease course (initial-onset, one month, three month and 6 month after ALA treatment). Top bars are maximum, bottom bars are minimum, the media bold bars are mean values of EDSS score of these 10 cases at the time points.
Figure $1 \mathrm{~B}$ is BOX analysis of EDSS scores from patients with inflammatory spine cords after the ALA treatment following the protocol. The whole box of each time point of EDSS shows the gradually decreasing through 6 months. The largest change of EDSS occurs at first month: Maximum changes from 7.5 to 6 , minimum changes from 1.5 to 0 . This result means that the patients with severest or lightest clinic disabilities have mostly improved in the first month. The biggest change of mean values of EDSS occurs between the first month and the third month, which means that most patients with mild clinic disorder have improved in three months. There are changes of EDSS after three months to six months, but it is not significant as earlier months after treatment. These results show a smaller improvement after third month of clinical symptoms but it is still very clear. The maintaining of administration of ALA is suggested.

Table 1 is a summary of EDSS scores with the Min, first quarter, Median, third quarter, Max and means. Over the whole treatment, the EDSS score decreases at a constant rate as time pass. Under initial condition, we have a score range from 1.500 to 7.500 with a mean of 4.350 and a median of 4.500 . After one month of treatment, we have a score range from 0.000 (meaning the patient has completely recovered) to 6.000 with a mean of 3.550 and a median of 4.250 . There was a 0.800 score difference in the means from the initial condition to the first month of treatment. However, there is only 0.250 score difference in the median. After three month of treatment, we have a score range from 0.000 to 5.500 with a mean of 2.701 and a median of 2.250 . The median decreased significantly since 1 st month of treatment. From this time, the median starts to score beneath the mean. After six month of treatment, we have a score range from 0.000 to 5.000 with a mean of 2.050 and a median of 1.750 . We do not see a significant difference between the scores at 3 rd months of treatment and 6th months of treatment.

\section{Discussion}

Inflammation in central nervous system (CNS) often results in severe disabilities through repeating collapse diseases course. Myelitis, Neuromylitis Optica (NMO) and Multiple Sclerosis (MS) are clinic common infectious diseases in spinal cord. The diseases course is that generally relapse following by a quiet period of remission that last months, even years without intervention therapies. ${ }^{1}$ Currently, the only effective routine therapeutic strategy is application of corticosteroid hormone in acute and outbreak phase in order to reduce the inflammation responses, which cause vascular damage, demyelination of neurons and formation of scar of CNS tissue. Interestingly, our previous study discovered that Dexamethasone (DXM), a corticosteroid hormone restores the neovascularization in spine cord of Experimental Autoimmune Encephalomyelitis (EAE), a MS mouse model..$^{10}$ Therefore, long-term/low dose of corticosteroid therapy has potential to improve the outcome of inflammation in CNS. However, clinical severe side effects limit its use.

ALA had been reported anti-oxidative stress role in degeneration diseases, metabolic disorder like diabetes. The remarkable discovery of ALA pharmacological roles are that reverse the age-related accumulation of iron in the rat cerebral cortex ${ }^{11}$ improve endothelial function, ${ }^{12}$ and increase insulin sensitivity in patients with diabetes, ${ }^{13}$ which imply ALA's intervention role in mitochondria. Therefore, ALA has potential capability to recover the function of Brain blood barrier (BBB) in injured CNS.

In this study, we provide the promising evidence that ALA treatment addition to routine corticosteroid therapy reverses inflammation legions, improves EDSS score, reduces frequency of relapse in this 10 cases acute and chronic infectious diseases of spinal cord. 


\section{Author contribution}

Guojun Tan designed experiments, performed clinical trial and collected data, and discussed the manuscript, provided funds for the study. Anne Hubbard analyzed data with statistics skill and generated the graphs and Table. Xin Wang analyzed data, edited figures and wrote the manuscript.

\section{Acknowledgements}

This work is supported by Natural Science Foundation of Hebei Province (Grant No: H2012206047).

\section{Conflict of interest}

The authors declare that the research is conducted in the absence of any commercial or financial relationships that could be constructed as a potential conflict of interest.

\section{Funding}

None.

\section{References}

1. Multiple Sclerosis-Diagnosis and treatment-Mayo Clinic.

2. Buchman AL. Side effects of corticosteroid therapy. J Clin Gastroenterol. 2001;33(4):289-294.

3. Alpha-Lipoic-Acid.

4. Sarezky D, Raquib AR, Dunaief JL, et al. Tolerability in the elderly population of high-dose alpha lipoic acid: a potential antioxidant therapy for the eye. Clinical Ophthalmology. 2016;10:1899-1903.
5. Singh U, Jialal Ishwarial. Alpha-lipoic acid supplementation and diabetes. Nutr Rev. 2008; 66(11):646-657.

6. Heitzer T, Finckh B, Albers S, et al. Beneficial effects of alpha-lipoic acid and ascorbic acid on endothelium-dependent, nitric oxide-mediated vasodilation in diabetic patients: relation to parameters of oxidative stress. Free Radic Biol Med. 2001;31(1):53-61.

7. Bast A, Haenen GR. Lipoic acid: a multifunctional antioxidant. Biofactors. 2003;17(1-4):207-213.

8. Yadav V, Marracci G, Lovera J, et al. Lipoic acid in multiple sclerosis: a pilot study. Mult Scler. 2005;11:159-165.

9. Plemel JR, Juzwik CA, Benson CA, et al. Over-the-counter anti-oxidant therapies for use in multiple sclerosis: A systematic review. Mult Scler. 2015;21(12):1485-1495.

10. Wang X, Zhang Y, Meng R, et al. Dexamethasone Restores the Neovascularization of Spine Cord in Experimental Autoimmune Encephalomyelitis (EAE) Rodent Models. J Cardiol Curr Res. 2015; 4(3):00142.

11. Suh JH, Moreau R, Heath SH, et al. Dietary supplementation with (R)-alpha-lipoic acid reverses the age-related accumulation of iron and depletion of antioxidants in the rat cerebral cortex. Redox Rep. 2005;10:52-60.

12. Sola S, Mir MQ, Cheema FA, et al. Irbesartan and lipoic acid improve endothelial function and reduce markers of inflammation in the metabolic syndrome: results of the Irbesartan and Lipoic Acid in Endothelial Dysfunction (ISLAND) study. Circulation. 2005;111(3):343-348.

13. Kamenova P. Improvement of insulin sensitivity in patients with type 2 diabetes mellitus after oral administration of alpha-lipoic acid. Hormones (Athens). 2006;5(4):251-258. 